1 Hacettepe Journal of Mathematics and Statistics

h Volume 46 (5) (2017), $801-815$

\title{
Shear horizontal waves in a nonlinear elastic layer overlying a rigid substratum
}

\author{
Dilek Demirkus* and Mevlut Teymur ${ }^{\dagger \dagger}$
}

\begin{abstract}
In this work, the propagation of shear horizontal ( $\mathrm{SH}$ ) waves in a homogeneous, isotropic and compressible nonlinear hyper-elastic layer having finite thickness is studied. The upper surface of the layer is assumed to be free from traction and the lower boundary is rigidly fixed. These waves are dispersive like the Love waves. The problem is examined by a perturbation method that balances the nonlinearity and dispersion in the analysis. A nonlinear Schrödinger equation is derived describing the nonlinear self modulation of the waves. Then, the effect of nonlinear properties of the material on the propagation characteristics and on the existence of solitary waves are discussed.
\end{abstract}

Keywords: Dispersive nonlinear shear horizontal waves, Multiple scale method, Bright solitary waves, Dark solitary waves.

2000 AMS Classification: AMS[2010], 74B20, 74J30, 74J35.

Received : 19.10.2016 Accepted : 06.12.2016 Doi : 10.15672/ HJMS.2017.426

\section{Introduction}

Elastic waves propagating in an unbounded media are non-dispersive i.e. phase velocities of waves are constants. On the other hand, in wave guides such as rods, plates, layered half space, etc.,the phase velocities of the waves depend on wave number, hence the waves are dispersive. Dispersive elastic waves have been studied extensively, because of their application in geophysics, nondestructive testing of materials,electronic signal processing devices, etc.. (see, e.g. Ewing et al. [1], Love [2], Achenbach [3], Graf [4], Farnell [5], Maugin [6]).

In recent years, the effect of the nonlinear material parameters on the propagation characteristics of dispersive elastic waves has been the subject of numerous investigations

*Istanbul Technical University, Department of Mathematics, 34469 Maslak, Istanbul, Turkey, Email: demirkusdilek@itu.edu.tr

$\dagger$ Istanbul Technical University, Department of Mathematics, 34469 Maslak, Istanbul, Turkey, Email: teymur@itu.edu.tr

$\ddagger$ Corresponding Author. 
for similar reasons mentioned above. By employing asymptotic perturbation methods many problems related with the propagation of nonlinear dispersive waves are examined. In these works, as a result of balance between nonlinearity and dispersion various nonlinear evaluation equations such as Korteweg-DeVries (KdV) equation, modified KortewegDeVries (mKdV) equation, nonlinear Schrödinger (NLS) equation, Boussinesq equation etc. have been derived to elucidate the nonlinear wave motion asymptotically. Then various aspects of the problems such as the stability of modulated wave, the existence of solitary waves, etc. were discussed on the basis of these equations. For an extensive review of most of these works we refer to Parker and Maugin [7], Maugin [8], Parker [9], Mayer [10], Norris [11], Porubov [12]. Among the works on nonlinear dispersive elastic waves, the investigations of nonlinear shear horizontal $(\mathrm{SH})$ waves occupies an important place. Below some of these works will be reviewed to relate the present work to them. In [13], Bataille and Lund considered the propagation of nonlinear Love waves in a layered half space covered by a thin linear elastic layer. A modified Boussinesq equation is derived by an intuitive approach guided by physical arguments which accounts the dispersive nature of Love waves and the nonlinearity. This equation has an approximate modulated solitary wave (an envelope solitary wave) solution which provides mechanisms for localized energy propagation along the surface of the layered medium. The propagation of nonlinear Love waves in a half space covered by a layer of uniform finite thickness having different mechanical properties, is investigated by a perturbation method in [14] by Teymur. The materials of the layer and the half space are both assumed to be homogeneous, isotropic and compressible hyper-elastic. Then, it is shown that the nonlinear self modulation of Love waves is governed asymptotically by an NLS equation. The coefficients of this NLS equation are valid on all branches of the linear dispersion relation of Love waves for any wave number. From the numerical evaluation of these coefficients for various material parameters it has been observed that the stability of modulated waves, the existence of envelope ( bright) and dark solitary waves depend strongly on the nonlinear properties of the layered media as well as the wave number. The problem is reconsidered by Maugin and Hadouaj [15] where the nonlinear substrate covered by a linear thin elastic layer and then by Teymur et al.[16] if the top layer is made of a thin nonlinear elastic material. In [17], Ahmetolan and Teymur studied the propagation of nonlinear SH waves and the formation of Love waves in a double layered plate each having finite thickness. In [18], Ahmetolan and Teymur examined the propagation of nonlinear SH waves in a plate having finite thickness and made of a generalized neo-Hookean material. An NLS equation is derived which governs the nonlinear self modulation of waves asymptotically and the effect of nonlinearity on the propagation characteristics is discussed. The propagation of small but finite amplitude long SH waves in a double layered plate is examined in [25] by Teymur . I! n that work by an asymptotic analysis, a modified KdV equation is derived and then the dependence of various types of solitary wave solutions on the nonlinear material parameters are discussed. The propagation of large amplitude Love waves in a layered half-space made of different pre-stressed compressible neo-Hookean materials (restricted Hadamard materials) is examined by Ferreira and Boluenger [20] and an exact solution of the problem is given. Later, the anti-plane shear motions coupled with an in-plane motion for a Hadamard materials are considered by Pucci and Saccomandi [21]. The pure anti-plane motion may be sustained in a Hadamard material in the absence of body forces. When the constitutive parameter $\beta$ is small, a perturbation analysis is developed using this small parameter. Then this approach is also applied to the propagation of finite amplitude Love waves in a layered half space made of Hadamard materials. And the solutions exhibiting a secondary in-plane motion caused by a principal anti-plane motion are given. 
In the present work, we consider the propagation $\mathrm{SH}$ waves in a homogeneous isotropic compressible nonlinear elastic finite layer deposited on a rigid substratum. The upper surface of the layer is assumed to be free from the stress. This problem may model some real world problem. A uniform layer of a nonlinear soil overlying a rigid bedrock is an example from soil dynamics (see for example[22]). Also a soft material layer overlying an almost rigid material is an another example from the signal processing applications (see for example [8] and [15]). The problem is examined by a perturbation method. By balancing the nonlinearity and dispersion in the analysis, an NLS equation is derived describing the nonlinear self modulation of $\mathrm{SH}$ waves. Then, the effect of nonlinearity on the propagation characteristics of waves and on the existence of solitary waves are discussed.

\section{Formulation of the Problem}

Let $\left(x_{1}, x_{2}, x_{3}\right)$ and $\left(X_{1}, X_{2}, X_{3}\right)$ be, respectively, the spatial and material coordinates of a point referred to the same rectangular Cartesian system of axes. Consider an elastic layer of uniform thickness $h$, occupying the regions between the planes $X_{2}=0$ and $X_{2}=h$ in the reference frame $X_{K}$. It is assumed that the boundary $X_{2}=h$ is free of traction and the displacements are zero at the rigid boundary $X_{2}=0$. Now, an SH wave described by the equations

$$
x_{k}=X_{K} \delta_{k K}+u_{3}\left(X_{\Delta}, t\right) \delta_{k 3}
$$

is supposed to propagate along the $X_{1}$-axis in the layer, where $u_{3}$ is the displacement of a particle in the $X_{3}$-direction, $t$ is the time and $\delta_{k K}$ is the Kronecker symbol. The summation convention on repeated indices is implied in (2.1) and in the sequel of this section, and Latin and Greek indices have respective ranges $(1,2,3)$ and $(1,2)$. Since $\operatorname{det}\left(\partial x_{k} / \partial X_{K}\right)=1$; the deformation field defined by $(2.1)$ is isochoric and the density $\rho$ of the layer in motion remain constant, i.e. $\rho=\rho_{0}=$ constant.

Let $T_{K l}$ be the first Piola-Kirchoff stress tensor field accompanying the deformation field (2.1); in the absence of body forces, the equation of motion in the reference state take the following forms

$$
T_{\Delta \beta, \Delta}+T_{3 \beta, 3}=0, \quad T_{\Delta 3, \Delta}+T_{33,3}=\rho_{0} \ddot{u}_{3}
$$

where subscripts preceded by a comma indicate partial differentiation with respect to coordinates $X_{K}$ and an over dot represents the partial differentiation with respect to $t$ [14].

The assumption of vanishing traction on the free surface of the layer imposes the boundary condition

$$
T_{2 k}=0 \quad \text { on } \quad X_{2}=h,
$$

and on the rigid boundary

$$
u_{3}=0 \quad \text { on } \quad X_{2}=0 .
$$

Let us now assume that the constituent material of the layer is nonlinear, homogeneous, isotropic and compressible hyper-elastic. Stress constitutive equations for such a material may be expressed as

$$
T_{K k}=\left(\frac{\partial \Sigma}{\partial I_{1}} \delta_{L K}+2 \frac{\partial \Sigma}{\partial I_{2}} E_{L K}+3 \frac{\partial \Sigma}{\partial I_{3}} E_{L M} E_{M K}\right) x_{k, L}
$$

where $E_{K L}=\left(x_{k, K} x_{k, L}-\delta_{K L}\right) / 2$ is the Lagrangian strain tensor and $\Sigma$ is the strain energy function (see e.g. Eringen and Suhubi [23]). For an isotropic material, $\Sigma$ is an isotropic function of the invariants of $\mathbf{E}$ defined as

$$
I_{1}=\operatorname{tr} \mathbf{E}, \quad I_{2}=\operatorname{tr} \mathbf{E}^{2}, \quad I_{3}=\operatorname{tr} \mathbf{E}^{3} .
$$


For the deformation field (2.1), the invariants are found to be

$$
I_{1}=Q / 2, \quad I_{2}=Q(1+Q / 2) / 2, \quad I_{3}=Q^{2}(3+Q) / 8 .
$$

where

$$
Q=Q\left(u_{3}\right)=u_{3, \Delta} u_{3, \Delta} .
$$

The stress-strain relations (2.5) now read [14],

$$
\begin{array}{r}
T_{\Delta \beta}=\left[\frac{\partial \Sigma}{\partial I_{1}} \delta_{\Omega \Delta}+\left(\frac{\partial \Sigma}{\partial I_{2}}+\frac{3}{4}(1+Q) \frac{\partial \Sigma}{\partial I_{3}}\right) u_{3, \Omega} u_{3, \Delta}\right] \delta_{\beta \Omega}, \quad T_{\Delta 3}=2 \frac{\partial \Sigma}{\partial Q} u_{3, \Delta}, \\
\text { (2.9) } \quad T_{3 \alpha}=-u_{3, \Delta} T_{\Delta \alpha}+\delta_{\alpha \Delta} T_{\Delta 3}, \quad T_{33}=\frac{\partial \Sigma}{\partial I_{1}}+\left(\frac{\partial \Sigma}{\partial I_{2}}+\frac{3}{4}(1+Q) \frac{\partial \Sigma}{\partial I_{3}}\right) Q .
\end{array}
$$

Note that for a specific material $\Sigma$ is a prescribed function of $Q$ through the invariants (2.7). Hence the equations of motion (2.2) are three equations to be satisfied by a single function $u_{3}$. For any material defined by (2.5), if the first two equations in (2.2) are satisfied by a given solution of the third equation in (2.2), then the motion (2.1) can exist in the medium in the absence of body forces. This is only the case if $\Sigma=\Sigma\left(I_{1}\right)$, i.e. if the medium is made of a generalized Neo-Hookean material ( see e.g. Teymur [19] or in more detail Saccomandi and Ogden [24] ). In general without any restriction on the constitutive relation (2.5) (or (2.9)), the system of equations (2.2) is not compatible so that the motion (2.1) cannot be maintained without body forces acting in the $\left(X_{1}, X_{2}\right)$-plane (see for details Carroll [25], Pucci and Saccomandi [26], Rogers et al. [27]). Therefore as in [14](or in [16]) we will assume that the motion (2.1) takes place in a material for which the Cauchy stress components $t_{\alpha \beta}$ are identically zero as in the case of linear problem. Hence, since $T_{\Delta \beta}=\delta_{\Delta \alpha} t_{\alpha \beta}$ then $T_{\Delta \beta}=0$ and as a consequence of this assumption, the first two equations in (2.2) are satisfied identically and the third equation becomes

$$
2\left(\frac{\partial \Sigma}{\partial Q} u_{3, \Delta}\right), \Delta=\rho_{0} \ddot{u}_{3} .
$$

It is seen from (2.9) that for such a material the strain energy function $\Sigma$ must satisfy the following conditions

$$
\frac{\partial \Sigma}{\partial I_{1}}=0, \quad \frac{\partial \Sigma}{\partial I_{2}}+\frac{3}{4}(1+Q) \frac{\partial \Sigma}{\partial I_{3}}=0,
$$

whenever the invariants are given by (2.7). We now employ the following fourth order polynomial expansion of $\Sigma$ in terms of the strain invariants $I_{i}$ to deduce approximate equations

$$
\begin{aligned}
& \Sigma=\alpha_{1} I_{1}^{2}+\alpha_{2} I_{2}+\alpha_{3} I_{1}^{3}+\alpha_{4} I_{1} I_{2}+\alpha_{5} I_{3}+\alpha_{6} I_{1}^{4}+\alpha_{7} I_{1}^{2} I_{2}+\alpha_{8} I_{1} I_{3}+\alpha_{9} I_{2}^{2} \\
& +O\left(I_{1}^{5}, I_{2}^{5 / 2}, I_{3}^{5 / 3}\right)
\end{aligned}
$$

where $\alpha_{1}=\lambda / 2, \alpha_{2}=\mu$ are second order ( $\lambda$ and $\mu$ are the usual Lamé constants), $\alpha_{3}, \alpha_{4}, \alpha_{5}$ are third order and $\alpha_{6}, \alpha_{7}, \alpha_{8}, \alpha_{9}$ are fourth-order elastic constants. The third order elastic constants related to the Murnaghan's constants $l, m, n$ as (see Norris [11])

$$
\alpha_{3}=\frac{1}{3}\left(l-m+\frac{1}{2} n\right) \quad, \alpha_{4}=\left(m-\frac{1}{2} n\right), \quad \alpha_{5}=\frac{n}{3}
$$

Then employing (2.12) in the stress-strain relation (2.9) and applying the restrictions on $\Sigma$ defined in (2.11) we get

$$
\begin{array}{r}
T_{11}=T_{12}=T_{21}=T_{22}=T_{33}=0, \\
T_{\Delta 3}=\mu u_{3, \Delta}+\left(\alpha_{9}+m / 2\right) u_{3, \Delta} Q+O\left(Q^{2}\right),
\end{array}
$$

and the equation (2.10) becomes

$$
\ddot{u}_{3}-c_{T}^{2} u_{3, \Delta \Delta}=n_{T}\left(u_{3, \Delta} Q\right), \Delta+O\left(Q^{2}\right)
$$


where

$$
c_{T}^{2}=\mu / \rho_{0}, \quad n_{T}=\left(\alpha_{9}+m / 2\right) / \rho_{0}
$$

Here, $c_{T}$ is the linear shear velocity and $n_{T}$ the nonlinear material constant which exhibit the nonlinear characteristics of the constituent material. When $n_{T}>0$, the medium is hardening in shear, but if $n_{T}<0$, then it is softening.

Hence, the SH wave motion (2.1) can be maintained in the restricted hyper-elastic material defined by (2.14) without body forces acting in the $\left(X_{1}, X_{2}\right)$ - plane. Now, let $X=X_{1}, Y=X_{2}, Z=X_{3}$ and $u=u_{3}$. Then from (2.14) and(2.15) the following approximate governing equation and boundary conditions involving terms not higher than the third degree in the deformation gradients are written;

$$
\begin{aligned}
& \frac{\partial^{2} u}{\partial t^{2}}-c_{T}^{2}\left(\frac{\partial^{2} u}{\partial X^{2}}+\frac{\partial^{2} u}{\partial Y^{2}}\right)=n_{T}\left[\frac{\partial}{\partial X}\left(\frac{\partial u}{\partial X} Q(u)\right)+\frac{\partial}{\partial Y}\left(\frac{\partial u}{\partial Y} Q(u)\right)\right] \\
& \frac{\partial u}{\partial Y}\left(1+\frac{n_{T}}{c_{T}^{2}} Q(u)\right)=0 \quad \text { on } Y=h, \\
& u=0 \quad \text { on } Y=0 \text {, }
\end{aligned}
$$

\section{Asymptotic analysis of the nonlinear SH waves}

In this work, how the slowly varying amplitude of a weakly nonlinear $\mathrm{SH}$ wave is modulated by nonlinear self interaction is investigated by a perturbation method. For this purpose, the method of the multiple scales is employed by introducing the following new independent variables

$$
x_{i}=\varepsilon^{i} X, \quad t_{i}=\varepsilon^{i} t, \quad y=Y ; \quad i=0,1,2, \ldots
$$

instead of $X, Y, t[28]$. Here $\varepsilon>0$ is a small parameter which measures the weakness of the nonlinearity, $\left\{x_{0}, t_{0}, y\right\}$ are fast variables describing the fast variations in the problem while $\left\{x_{1}, x_{2}, \ldots, t_{1}, t_{2}, \ldots\right\}$ are slow variables describing the slow variations. Now, $u$ is considered to be a function of these new variables and it is expanded in the following asymptotic series in $\varepsilon$;

$$
u=\sum_{n=1}^{\infty} \varepsilon^{n} u_{n}\left(x_{0}, x_{1}, x_{2}, \ldots, y, t_{0}, t_{1}, t_{2}, \ldots\right)
$$

In this work we aimed to obtain first order uniformly valid asymptotic solution of the problem. Therefore in the following part we will assume the dependence of $u_{n}$ on the slow scales $\left\{x_{1}, x_{2}, t_{1}, t_{2}\right\}$ only. If one studies the contribution of higher order terms then the third order, in the analysis the dependence on the slower scales $\left\{x_{3}, \ldots, t_{3}, \ldots\right\}$ should also be considered as independent variables. Now, first writing the equation of motion (2.17), the boundary conditions (2.18) and (2.19) in terms of the new independent variables (3.1) and then employing the asymptotic expansion (3.2) in the resulting expressions and collecting the terms of like powers of in $\varepsilon$, we obtain a hierarchy of problems from which it is possible to determine $u_{n}$, successively. Up to third order in $\varepsilon$ these are given as follows;

$\mathcal{O}(\varepsilon):$

$$
\begin{aligned}
\mathcal{L} u_{1} & \triangleq \frac{\partial^{2} u_{1}}{\partial t_{0}^{2}}-c_{T}^{2}\left(\frac{\partial^{2} u_{1}}{\partial x_{0}^{2}}+\frac{\partial^{2} u_{1}}{\partial y^{2}}\right)=0, \\
\frac{\partial u_{1}}{\partial y} & =0 \quad \text { on } \quad y=h, \\
u_{1} & =0 \quad \text { on } \quad y=0 .
\end{aligned}
$$


$\mathcal{O}\left(\varepsilon^{2}\right):$

$$
\begin{aligned}
& \mathcal{L} u_{2}=2\left(c_{T}^{2} \frac{\partial^{2} u_{1}}{\partial x_{0} \partial x_{1}}-\frac{\partial^{2} u_{1}}{\partial t_{0} \partial t_{1}}\right), \\
& \frac{\partial u_{2}}{\partial y}=0 \quad \text { on } \quad y=h, \\
& u_{2}=0 \quad \text { on } \quad y=0 . \\
& \mathcal{L} u_{3}=2\left(c_{T}^{2} \frac{\partial^{2} u_{2}}{\partial x_{0} \partial x_{1}}-\frac{\partial^{2} u_{2}}{\partial t_{0} \partial t_{1}}\right)+c_{T}^{2}\left(\frac{\partial^{2} u_{1}}{\partial x_{1}^{2}}+2 \frac{\partial^{2} u_{1}}{\partial x_{0} \partial x_{2}}\right)-\frac{\partial^{2} u_{1}}{\partial t_{1}^{2}}-2 \frac{\partial^{2} u_{1}}{\partial t_{0} \partial t_{2}} \\
&+n_{T} {\left[\frac{\partial}{\partial x_{0}}\left(\frac{\partial u_{1}}{\partial x_{0}} \mathcal{K}\left(u_{1}\right)\right)+\frac{\partial}{\partial y}\left(\frac{\partial u_{1}}{\partial y} \mathcal{K}\left(u_{1}\right)\right)\right] } \\
& \frac{\partial u_{3}}{\partial y}+\frac{n_{T}}{c_{T}^{2}} \mathcal{K}\left(u_{1}\right) \frac{\partial u_{1}}{\partial y}=0 \quad \text { on } \quad y=h \\
& u_{3}=0 \quad \text { on } y=0
\end{aligned}
$$

where

$$
\mathcal{K}\left(u_{1}\right)=\left(\frac{\partial u_{1}}{\partial x_{0}}\right)^{2}+\left(\frac{\partial u_{1}}{\partial y}\right)^{2}
$$

Note that the perturbation problems are linear in each step. Moreover the first order problem is simply the classical linear problem which was first investigated by Hudson [29]. Let us examine this problem. For the existence of an SH wave in the layer, the phase velocity of the wave must satisfy the inequality

$$
c>c_{T}
$$

i.e. an SH wave having a phase velocity less than the linear shear wave velocity $c_{T}$ of the medium does not propagate in the layer. We proceed by assuming that the above inequality is satisfied by the phase velocity of the $\mathrm{SH}$ wave. Then by using the separation of variables method, the solution of the governing equation (3.3) is found to be

$$
u_{1}=\sum_{\ell=1}^{\infty}\left[A_{1}^{(\ell)}\left(x_{1}, x_{2}, t_{1}, t_{2}\right) e^{i \ell k p y}+B_{1}^{(\ell)}\left(x_{1}, x_{2}, t_{1}, t_{2}\right) e^{-i \ell k p y}\right] e^{i \ell \phi}+\text { c.c. }
$$

where

$$
p=\left(c^{2} / c_{T}^{2}-1\right)^{1 / 2}, \quad \phi=k x_{0}-\omega t_{0} .
$$

and $A_{1}^{(\ell)}, B_{1}^{(\ell)}$ are the first order amplitude functions of the slow variables, $k$ is the wave number, $\omega$ is the angular frequency, $c=\omega / k$ is the phase velocity, and c.c. denotes the complex conjugate to the preceding terms. The substitution (3.14) into the boundary conditions (3.4) and (3.5) yields the following system of homogeneous linear equations for the first order amplitude functions;

$$
\mathbf{W}_{\ell} \mathbf{U}_{1}^{(\ell)}=\mathbf{0}
$$

where

$$
\mathbf{W}_{\ell}=\left[\begin{array}{cc}
i \ell k p e^{i \ell k p h} & -i \ell k p e^{-i \ell k p h} \\
1 & 1
\end{array}\right]
$$

is the dispersion matrix and, $\mathbf{U}_{1}^{(\ell)}$ are the first order amplitude vectors defined as

$$
\mathbf{U}_{1}^{(\ell)}=\left[\begin{array}{c}
A_{1}^{(\ell)} \\
B_{1}^{(\ell)}
\end{array}\right] \text {. }
$$


Note that $\operatorname{det} \mathbf{W}_{1}=0$ gives the dispersion relation of the linear $\mathrm{SH}$ waves [29];

$$
\cos (k p h)=0 \text {. }
$$

Hence

$$
k p h=(2 n-1) \frac{\pi}{2}, \quad \text { for } \quad \mathrm{n}=1,2, \ldots
$$

From here we write

$$
\frac{c^{2}}{c_{T}^{2}}=1+\left[\frac{(2 n-1) \pi}{2 k h}\right]^{2} \quad \text { or } \quad \omega^{2}=k^{2} c_{T}^{2}\left\{1+\left[\frac{(2 n-1) \pi}{2 k h}\right]^{2}\right\}
$$

where $n$ denotes the branches of the linear dispersion relation $\omega=\omega(k ; n)$. Note that the dispersion relation (3.19) (or (3.21)) is the same of the dispersion relation for the antisymmetric motion of SH waves in an elastic isotropic plate with the thickness $2 h$ occupying the region between the planes $Y=h$ and $Y=-h$. Therefore, the displacement field also corresponds to the antisymmetric deformation of the plate in the half part $[h, 0]$ (see [4] or [18]). Since the purpose of this paper is to examine the nonlinear self modulation of waves centered around a wave number $k$, with corresponding frequency $\omega$. To exclude the harmonic-resonance in the analysis, we have to assume that

$$
\operatorname{det} \mathbf{W}_{\ell} \neq 0 \quad \text { for } \quad \ell \neq 1 \text {. }
$$

Then the solutions of the system of linear equations (3.16) are found to be

$$
\mathbf{U}_{1}^{(1)}=\mathscr{A}_{1}\left(x_{1}, x_{2}, t_{1}, t_{2}\right) \mathbf{R}
$$

and

$$
\mathbf{U}_{1}^{(\ell)}=\mathbf{0} \quad \text { for } \quad \ell \geq 2
$$

where $\mathscr{A}_{1}$ is a complex function of the slow variables describing slowly varying amplitude of wave modulation, and $\mathbf{R}$ is a column vector satisfying

$$
\mathbf{W}_{1} \mathbf{R}=\mathbf{0}
$$

Hence $\mathbf{R}$ can be taken as

$$
\mathbf{R}=\left[\begin{array}{l}
R_{1} \\
R_{2}
\end{array}\right]=\left[\begin{array}{c}
1 \\
e^{2 i k p h}
\end{array}\right]
$$

and the first order solution is written explicitly as

$$
u_{1}=\mathscr{A}_{1}\left(x_{1}, x_{2}, t_{1}, t_{2}\right)[2 i \sin (k p y)] e^{i \phi}+\text { c.c. }
$$

Note that, the first order solution given in (3.27) and the solution of the linear problem are of the same form(see [29]). The only difference is that, in the linear problem $\mathscr{A}_{1}$ is a constant, but here it is a slowly varying function of the slow variables representing the nonlinear self-modulation of a wave train. To complete the first order solution of the nonlinear problem this function has to be determined. Therefore, to achieve this we proceed to examine the higher order perturbation problems. The use of the first order solution (3.27) in (3.6) of the second order perturbation problem yields

$$
\mathcal{L} u_{2}=2 i\left(\omega \frac{\partial \mathscr{A}_{1}}{\partial t_{1}}+k c_{T}^{2} \frac{\partial \mathscr{A}_{1}}{\partial x_{1}}\right)\left(e^{i k p y}+e^{i k p(2 h-y)}\right) e^{i \phi}+\text { c.c. }
$$

We now decompose the solution of (3.28) as

$$
u_{2}=\bar{u}_{2}+\tilde{u}_{2}
$$


where $\bar{u}_{2}$ is the particular solution of non-homogeneous equation (3.28) and $\tilde{u}_{2}$ denotes the solution of the following problem obtained from the second order problem by the use of the decomposition (3.29)

$$
\begin{array}{ll}
\mathcal{L} \tilde{u}_{2}=0 & \\
y=0 ; & \tilde{u}_{2}=-\bar{u}_{2} \\
y=h ; & \frac{\partial \tilde{u}_{2}}{\partial y}=-\frac{\partial \bar{u}_{2}}{\partial y}
\end{array}
$$

The particular solution $\bar{u}_{2}$ of the non-homogeneous equation (3.28) is found by the method of undetermined coefficient as

$$
\bar{u}_{2}=\frac{1}{k p c_{T}^{2}}\left(\omega \frac{\partial \mathscr{A}_{1}}{\partial t_{1}}+k c_{T}^{2} \frac{\partial \mathscr{A}_{1}}{\partial x_{1}}\right)\left(-e^{i k p y}+e^{i k p(2 h-y)}\right) y e^{i \phi}+\text { c.c. }
$$

The solution $\tilde{u}_{2}$ of the homogeneous equation (3.30), as in the first order problem, can be written as follows

$$
\tilde{u}_{2}=\sum_{\ell=1}^{\infty}\left[A_{2}^{(\ell)}\left(x_{1}, x_{2}, t_{1}, t_{2}\right) e^{i \ell k p y}+B_{2}^{(\ell)}\left(x_{1}, x_{2}, t_{1}, t_{2}\right) e^{-i \ell k p y}\right] e^{i \ell \phi}+\text { c.c. }
$$

where $A_{2}^{(\ell)}$ and $B_{2}^{(\ell)}$ are the second order amplitude functions of the slow variables. Then the use of this solution together with the particular solution (3.33) in the boundary conditions (3.32) and (3.33), yields the following linear system of equations to determine $A_{2}^{(\ell)}$ and $B_{2}^{(\ell)}$

$$
\mathbf{W}_{\ell} \mathbf{U}_{2}^{(\ell)}=\mathbf{b}_{2}^{(\ell)}
$$

where

$$
\mathbf{U}_{2}^{(\ell)}=\left[\begin{array}{c}
A_{2}^{(\ell)} \\
B_{2}^{(\ell)}
\end{array}\right]
$$

and the vectors $\mathbf{b}_{2}^{(\ell)}$ are found as

$$
\mathbf{b}_{2}^{(1)}=\left[\frac{2 i h}{c_{T}^{2}}\left(\omega \frac{\partial \mathscr{A}_{1}}{\partial t_{1}}+k c_{T}^{2} \frac{\partial \mathscr{A}_{1}}{\partial x_{1}}\right) e^{i k p h}\right]
$$

and

$$
\mathbf{b}_{2}^{(\ell)}=\mathbf{0} \quad \text { for } \quad \ell \geq 2
$$

A little algebra reveals that, $\mathbf{b}_{2}^{(1)}$ can be put into the following form

$$
\mathbf{b}_{2}^{(1)}=-i\left(\frac{\partial \mathscr{A}_{1}}{\partial t_{1}} \frac{\partial \mathbf{W}_{1}}{\partial \omega}-\frac{\partial \mathscr{A}_{1}}{\partial x_{1}} \frac{\partial \mathbf{W}_{1}}{\partial k}\right) \mathbf{R}
$$

For $\ell \neq 1$, since it is assumed that $\operatorname{det} \mathbf{W}_{\ell} \neq 0$ for $\ell \neq 1$, the solutions of (3.35) are

$$
\mathbf{U}_{2}^{(\ell)} \equiv \mathbf{0}, \quad \ell \neq 1
$$

but, since $\operatorname{det} \mathbf{W}_{1}=0$ and $\mathbf{b}_{2}^{(1)} \neq \mathbf{0}$, in order that the linear system of equations (3.35) solvable for $\mathbf{U}_{2}^{(1)}$, the compatibility condition

$$
\mathbf{L} \cdot \mathbf{b}_{2}^{(1)}=0
$$

must be satisfied, where $\mathbf{L}$ is a row vector defined by

(3.42) $\quad \mathbf{L W}_{1}=\mathbf{0}$

and an $\mathbf{L}$ can be taken as

$$
\mathbf{L}=\left[L_{1}, L_{2}\right]=\left[i, k p e^{i k p h}\right]
$$


The compatibility condition (3.41) then yields

$$
\frac{\partial \mathscr{A}_{1}}{\partial t_{1}}+V_{g} \frac{\partial \mathscr{A}_{1}}{\partial x_{1}}=0
$$

where $V_{g}$ is the group velocity of the waves defined as

$$
V_{g}=\frac{d \omega}{d k}=-\left(\mathbf{L} \frac{\partial \mathbf{W}_{1}}{\partial k} \mathbf{R}\right) /\left(\mathbf{L} \frac{\partial \mathbf{W}_{1}}{\partial \omega} \mathbf{R}\right)
$$

This equation state that the first order amplitude $\mathscr{A}_{1}$ remains constant in a frame of reference moving with the group velocity $V_{g}$, i.e. $\mathscr{A}_{1}=\mathscr{A}_{1}\left(x_{1}-V_{g} t_{1}, x_{2}, t_{2}\right)$. Then the solution of the equation (3.35) for $\ell=1$ can be written as

$$
\mathbf{U}_{2}^{(1)}=\mathscr{A}_{2} \mathbf{R}-i \frac{\partial \mathscr{A}_{1}}{\partial x_{1}}\left(\frac{\partial \mathbf{R}}{\partial k}+V_{g} \frac{\partial \mathbf{R}}{\partial \omega}\right)
$$

where the complex function $\mathscr{A}_{2}$ is the second order amplitude and is a function of slow variables, and it remains arbitrary in this order. It can be calculated in higher-order problems when necessary. But, since this work is centered around the propagation of weakly non-linear waves it is aimed to obtain just uniformly valid first order solution. To obtain the first order solution, $\mathscr{A}_{2}$ need not to be calculated explicitly, the determination of $\mathscr{A}_{1}$ will be sufficient and it will be done at the third order problem. Note that, if we assume that $\mathscr{A}_{2}$ depends on $x_{1}$ and $t_{1}$ through the combination $x_{1}-V_{g} t_{1}$ as $\mathscr{A}_{1}$, we can absorb it into $\mathscr{A}_{1}$ since it is proportional to $e^{i \phi}$ as $\mathscr{A}_{1}$. Therefore in the following calculations we omit $\mathscr{A}_{2}$. Now the substitution of the first and second order solutions into the third order equation (3.9) yields

$$
\begin{array}{r}
\mathcal{L} u_{3}=\left[\left(\mathscr{C}_{1}+\mathscr{C}_{2} y\right) e^{i k p y}+\left(\mathscr{C}_{3}+\mathscr{C}_{4} y\right) e^{-i k p y}+\mathscr{C}_{5} e^{3 i k p y}+\mathscr{C}_{6} e^{-3 i k p y}\right] e^{i \phi} \\
+ \text { c.c. }+ \text { terms in }\left(e^{ \pm 3 i \phi}\right)
\end{array}
$$

where

$$
\begin{gathered}
\mathscr{C}_{1}=2 i\left(\omega \frac{\partial \mathscr{A}_{1}}{\partial t_{2}}+k c_{T}^{2} \frac{\partial \mathscr{A}_{1}}{\partial x_{2}}\right)+c_{T}^{2} \frac{\partial^{2} \mathscr{A}_{1}}{\partial x_{1}^{2}}-\frac{\partial^{2} \mathscr{A}_{1}}{\partial t_{1}^{2}}-n_{T} k^{4}\left(9 p^{4}+2 p^{2}+9\right) \mathscr{A}_{1}\left|\mathscr{A}_{1}\right|^{2}, \\
\mathscr{C}_{2}=\left(-2 i / k p c_{T}^{2}\right)\left(\omega^{2} \frac{\partial^{2} \mathscr{A}_{1}}{\partial t_{1}^{2}}+2 \omega k c_{T}^{2} \frac{\partial^{2} \mathscr{A}_{1}}{\partial t_{1} \partial x_{1}}+k^{2} c_{T}^{4} \frac{\partial^{2} \mathscr{A}_{1}}{\partial x_{1}^{2}}\right), \quad \mathscr{C}_{3}=\mathscr{C}_{1} e^{2 i k h p}, \\
\mathscr{C}_{4}=-\mathscr{C}_{2} e^{2 i k h p}, \quad \mathscr{C}_{5}=n_{T} k^{4}\left(9 p^{4}-2 p^{2}-3\right) e^{-2 i k h p} \mathscr{A}_{1}\left|\mathscr{A}_{1}\right|^{2}, \\
\mathscr{C}_{6}=n_{T} k^{4}\left(9 p^{4}-2 p^{2}-3\right) e^{4 i k h p} \mathscr{A}_{1}\left|\mathscr{A}_{1}\right|^{2} .
\end{gathered}
$$

Now, as in the second order problem we decompose $u_{3}$ as

$$
u_{3}=\bar{u}_{3}+\tilde{u}_{3}
$$

where $\bar{u}_{3}$ denotes the particular solution of the equation (3.47) and $\tilde{u}_{3}$ denotes the solution of the following problem

$$
\begin{aligned}
& \mathcal{L} \tilde{u}_{3}=0 \\
& \tilde{u}_{3}=-\bar{u}_{3} \quad \text { on } \quad y=0, \\
& \frac{\partial \tilde{u}_{3}}{\partial y}=-\frac{\partial \bar{u}_{3}}{\partial y}-\frac{n_{T}}{c_{T}^{2}} \mathcal{K}\left(u_{1}\right) \frac{\partial u_{1}}{\partial y} \quad \text { on } \quad y=h .
\end{aligned}
$$

The particular solution $\bar{u}_{3}$ of the equation (3.47) can be expressed as a sum of linearly independent terms of the form

$$
\bar{u}_{3}=f_{3}^{(1)}\left(x_{1}, x_{2}, y, t_{1}, t_{2}\right) e^{i \phi}+f_{3}^{(3)}\left(x_{1}, x_{2}, y, t_{1}, t_{2}\right) e^{3 i \phi}+\text { c.c. },
$$

where the terms $f_{3}^{(1)}$ and $f_{3}^{(3)}$ represent the self interaction and the third harmonic interaction of the waves, respectively.In this work since only the self interaction is considered, in the sequel the explicit form of the term $f_{3}^{(3)}$ will not be required. Therefore, only $f_{3}^{(1)}$ 
will be calculated and this solution is obtained by the method of undetermined coefficient as

$$
f_{3}^{(1)}=\left(\mathscr{D}_{1}+\mathscr{D}_{2} y\right) y e^{i k p y}+\left(\mathscr{D}_{3}+\mathscr{D}_{4} y\right) y e^{-i k p y}+\mathscr{D}_{5} e^{3 i k p y}+\mathscr{D}_{6} e^{-3 i k p y}
$$

where

$$
\begin{array}{r}
\mathscr{D}_{1}=i \mathscr{C}_{1} / 2 k p c_{T}^{2}-\mathscr{C}_{2} / 4 k^{2} p^{2} c_{T}^{2}, \quad \mathscr{D}_{2}=i \mathscr{C}_{2} / 4 k p c_{T}^{2}, \\
\mathscr{D}_{3}=-i \mathscr{C}_{3} / 2 k p c_{T}^{2}-\mathscr{C}_{4} / 4 k^{2} p^{2} c_{T}^{2}, \quad \mathscr{D}_{4}=-i \mathscr{C}_{4} / 4 k p c_{T}^{2}, \\
\mathscr{D}_{5}=\mathscr{C}_{5} / 8 k^{2} p^{2} c_{T}^{2}, \quad \mathscr{D}_{6}=\mathscr{C}_{6} / 8 k^{2} p^{2} c_{T}^{2} .
\end{array}
$$

The problem posed for $\tilde{u}_{3}$ can be treated as in the second order problem. The solution of the homogeneous equation (3.50) therefore can be written as

$$
\tilde{u}_{3}=\sum_{\ell=1}^{\infty}\left[A_{3}^{(\ell)}\left(x_{1}, x_{2}, t_{1}, t_{2}\right) e^{i \ell k p y}+B_{3}^{(\ell)}\left(x_{1}, x_{2}, t_{1}, t_{2}\right) e^{-i \ell k p y}\right] e^{i \ell \phi}+\text { c.c. }
$$

where $A_{3}^{(\ell)}$ and $B_{3}^{(\ell)}$ are the third order slowly varying amplitude functions. Then the use of this solution together with the solutions $u_{1}, u_{2}$ and $\bar{u}_{3}$ in the boundary conditions (3.51)-(3.52) yields the following linear system of equations to determine $A_{3}^{(\ell)}$ and $B_{3}^{(\ell)}$;

$$
\mathbf{W}_{\ell} \mathbf{U}_{3}^{(\ell)}=\mathbf{b}_{3}^{(\ell)}
$$

where

$$
\mathbf{b}_{3}^{(1)} \neq \mathbf{0}, \quad \mathbf{b}_{3}^{(3)} \neq \mathbf{0}, \quad \text { and } \quad \mathbf{b}_{3}^{(\ell)}=\mathbf{0} \quad \text { for all } \ell \neq 1,3 .
$$

A lengthy but straight forward calculation discloses that $\mathbf{b}_{3}^{(1)}$ can be expressed as in the following form

$$
\begin{aligned}
& \mathbf{b}_{3}^{(1)}=-i\left(\frac{\partial \mathbf{W}_{1}}{\partial \omega} \frac{\partial \mathscr{A}_{1}}{\partial t_{2}}-\frac{\partial \mathbf{W}_{1}}{\partial k} \frac{\partial \mathscr{A}_{1}}{\partial x_{2}}\right) \mathbf{R} \\
& +\frac{1}{2}\left(\frac{\partial^{2} \mathbf{W}_{1}}{\partial \omega^{2}} \frac{\partial^{2} \mathscr{A}_{1}}{\partial t_{1}^{2}}-2 \frac{\partial^{2} \mathbf{W}_{1}}{\partial \omega \partial} \frac{\partial^{2} \mathscr{A}_{1}}{\partial x_{1} \partial t_{1}}+\frac{\partial^{2} \mathbf{W}_{1}}{\partial k^{2}} \frac{\partial^{2} \mathscr{A}_{1}}{\partial x_{1}^{2}}\right) \mathbf{R} \\
& +\left(\frac{\partial \mathbf{W}_{1}}{\partial k} \frac{\partial^{2} \mathscr{A}_{1}}{\partial x_{1}^{2}}-\frac{\partial \mathbf{W}_{1}}{\partial \omega} \frac{\partial^{2} \mathscr{A}_{1}}{\partial x_{1} \partial t_{1}}\right)\left(\frac{\partial \mathbf{R}}{\partial k}+V_{g} \frac{\partial \mathbf{R}}{\partial \omega}\right)+\mathbf{F}\left|\mathscr{A}_{1}\right|^{2} \mathscr{A}_{1}
\end{aligned}
$$

where the components of the vector $\mathbf{F}$ are found to be

$$
F_{1}=-i\left[\frac{n_{T} k^{4} h}{c_{T}^{2}}\left(9 p^{4}+2 p^{2}+9\right) \sin (k p h)\right], \quad F_{2}=0
$$

The explicit form of the vector $\mathbf{b}_{3}^{(3)}$ is not given here, since it represents the third harmonic interactions and therefore in the sequel it will not be required. For $\ell=1,(3.57)$ is an inhomogeneous equation and since $\operatorname{det} \mathbf{W}_{1}=0$, in order that this equation be solvable for $\mathbf{U}_{3}^{(1)}$ the following compatibility condition

(3.61) $\quad \mathbf{L} \cdot \mathbf{b}_{3}^{(1)}=0$

must be satisfied. For $\ell=3,(3.57)$ is an inhomogeneous equation, but since it is assumed that $\operatorname{det} \mathbf{W}_{3} \neq 0$ then the solution of (3.57) for this case is written as

$$
\mathbf{U}_{3}^{(3)}=\mathbf{W}_{3}^{-1} \mathbf{b}_{3}^{(3)}
$$

When $\ell \neq 1,3,(3.57)$ is a homogeneous equation and since $\operatorname{det} \mathbf{W}_{\ell} \neq 0$ for $\ell \neq 1$ by assumption, then the solutions are

$$
\mathbf{U}_{3}^{(\ell)}=\mathbf{0}
$$


An attempt will not be made towards obtaining the third order solutions explicitly since there will be no need for their explicit forms. The analysis will be continued by the examination of the solvability condition (3.61) to be satisfied at this order. This condition yields the following equations for $\mathscr{A}_{1}$;

$$
i\left(\frac{\partial \mathscr{A}_{1}}{\partial t_{2}}+V_{g} \frac{\partial \mathscr{A}_{1}}{\partial x_{2}}\right)+\tilde{\Gamma} \frac{\partial^{2} \mathscr{A}_{1}}{\partial x_{1}^{2}}+\tilde{\Delta}\left|\mathscr{A}_{1}\right|^{2} \mathscr{A}_{1}=0
$$

where

$$
\tilde{\Gamma}=\frac{1}{2} \frac{d V_{g}}{d k}=\frac{1}{2} \frac{d^{2} \omega}{d k^{2}}, \quad \tilde{\Delta}=-\mathbf{L} \cdot \mathbf{F} /\left(\mathbf{L} \frac{\partial \mathbf{W}_{1}}{\partial \omega} \mathbf{R}\right)
$$

In terms of the following non-dimensional variables and constants

$$
\tau=\omega t_{2}, \quad \xi=k\left(x_{1}-V_{g} t_{1}\right), \quad \mathscr{A}=k \mathscr{A}_{1}, \quad \Gamma=k^{2} \tilde{\Gamma} / \omega, \quad \Delta=\tilde{\Delta} / \omega k^{2}
$$

this equation can be rewritten in the standard NLS equation form as

$$
i \frac{\partial \mathscr{A}}{\partial \tau}+\Gamma \frac{\partial^{2} \mathscr{A}}{\partial \xi^{2}}+\Delta|\mathscr{A}|^{2} \mathscr{A}=0
$$

Thus, once a solution for $\mathscr{A}$ is derived from (3.67) for a given initial value of the form

$$
\mathscr{A}(\xi, 0)=\mathscr{A}_{0}(\xi)
$$

then the first order solution $u_{1}$ can be constructed by (3.27). Hence our task is completed. Here,it is obvious that the initial value $\mathscr{A}_{0}$ is related to the initial values of the first order displacement in the layer via (3.27).

\section{Concluding remarks}

We now examine the coefficients $\Gamma$ and $\Delta$ of the NLS equation obtained in this work, so that solutions of this equation are effected strongly by the sign of the product $\Gamma \Delta$. From (3.65) and (3.66) it is found that

$$
\Gamma \Delta=-n_{T} \frac{p^{2} c_{T}^{4}}{4 c^{6}}\left(9 p^{4}+2 p^{2}+9\right)
$$

Since

$$
\frac{p^{2} c_{T}^{4}}{4 c^{6}}\left(9 p^{4}+2 p^{2}+9\right)>0
$$

for all phase velocities $c>c_{T}$, then it is seen that if $n_{T}<0$, i.e. if the layer is made of a softening material, then $\Gamma \Delta>0$ for all phase velocities $c>c_{T}$. But if $n_{T}>0$,i.e if the layer is made of a hardening material, then $\Gamma \Delta<0$ for all phase velocities $c>c_{T}$. The variation of $C, V_{g}, \Gamma, \Delta$, and $\Gamma \Delta$ with the non-dimensional wave number $K=k h$ for the first three branches of the dispersion relation (3.21) are calculated and they are plotted in Fig(1), Fig(2), and Fig(3) respectively.

The NLS equation (3.67), as in this work, asymptotically describes the self modulation of the monochromatic plane waves in a nonlinear dispersive medium[30, 31]. It is also well known that the criterion whether $\Gamma \Delta>0$ or $\Gamma \Delta<0$ is important in determining how a given initial data will evolve for long times for the asymptotic wave field governed by the NLS equation. An initial disturbance vanishing as $|\xi| \rightarrow \infty$ tends to become a series of envelope solitary waves if $\Gamma \Delta>0$, while it evolves into decaying oscillations if $\Gamma \Delta<0$. On the other hand for disturbances that tend to a uniform state at infinity the envelope dark solitons exist for $\Gamma \Delta<0[30,31]$. The behavior of the traveling wave solutions of the NLS equation of the form

$$
\mathscr{A}(\xi, \tau)=\phi(\eta) e^{i(K \xi-\Omega \tau)}, \quad \eta=\xi-V_{0} \tau, \quad V_{0}=\text { constant }
$$


also depend on the sign of $\Gamma \Delta$. For $\Gamma \Delta>0$, if $\phi \rightarrow 0$ and $d \phi / d \eta \rightarrow 0$ as $|\eta| \rightarrow \infty$ the solution for $\phi$ is

(4.4) $\phi(\eta)=\phi_{0} \operatorname{sech}\left[(\Delta / 2 \Gamma)^{1 / 2} \phi_{0} \eta\right], \quad V_{0}=2 K \Gamma$

where $\left(\Gamma K^{2}-\Omega\right) / \Delta \phi_{0}^{2}=1 / 2$. This solution is known as envelope soliton or bright soliton [30, 31, 32]. When $\Gamma \Delta<0$ and $\left(\Gamma K^{2}-\Omega\right) / \Delta \phi_{0}^{2}=1$, if $\phi \rightarrow \phi_{0}$ and $d \phi / d \eta \rightarrow 0$ as $|\eta| \rightarrow \infty$, the solution for $\phi$ is

$$
\phi(\eta)=\phi_{0} \tanh \left[(-\Delta / 2 \Gamma)^{1 / 2} \phi_{0} \eta\right], \quad V_{0}=2 K \Gamma
$$

which represents the propagation of a phase jump [30, 31, 32]. Also, when $\Gamma \Delta<0$ there are no solutions of the NLS equation (3.67) corresponding to the envelope soliton solution (4.5) of the case $\Gamma \Delta>0$. However, a solution of the form

$$
\mathscr{A}(\xi, \tau)=\phi(\eta) e^{i\left[\Gamma^{2} \Delta \phi_{0}^{2} \tau-F(\eta)\right]}
$$

which tends to the uniform solution $\phi_{0} e^{i \Gamma^{2} \Delta \phi_{0}^{2} \tau}$ as $|\eta| \rightarrow \infty$ exists, where

$$
\phi^{2}=\phi_{0}^{2}\left(1-\sin ^{2} B \operatorname{sech}^{2} \psi\right), \quad F=\arctan (\tan B \tanh \psi)
$$

In (4.8) $B$ is a constant, $\psi$ and $V_{0}$ are given as

$$
\psi=(-\Gamma \Delta / 2)^{1 / 2} \phi_{0} \eta \sin B, \quad V_{0}= \pm 2^{-3 / 2} \Gamma(-\Gamma \Delta)^{1 / 2} \phi_{0}
$$

This solution is known as dark soliton and it has all the usual soliton features [32]. The NLS equation (3.67) has also plane wave solutions whether $\Gamma \Delta>0$ or $\Gamma \Delta<0$. Hence, by considering the above given short review about the effect of the sign of $\Gamma \Delta$ on the properties of the solution of an NLS equation, we conclude that when the layer is made of a softening material, since $\Gamma \Delta>0$ for all $k h>0$ in this case, the envelope solitary $\mathrm{SH}$ waves will exist and propagate in such a medium. But, when the plate is made of a hardening material since $\Gamma \Delta<0$ for all $k h>0$ in this case, then only the dark solitary $\mathrm{SH}$ waves will exist in such a layer. The modulated envelope solitary waves (4.4) existing in a softening elastic layer may provide mechanisms for an efficient energy propagation along the free surface of the layer. Several investigator have shown that the shear stress is a nonlinear function of the strain in certain soils and $n_{T}<0$, that is the response of the soil is softening in shear (see e.g.[33] and references given there). Therefore, when we consider a finite soil layer deposited on a rigid bedrock and showing this behavior under dynamic loading; one can observe the existence of an envelope soliton. An experimental result about the observation of solitons in soil mechanics was reported in [34] by Dimitriu.

\section{Acknowledgment}

We Would like to thank the referee for the invaluable comments leading to improvements to this paper. 


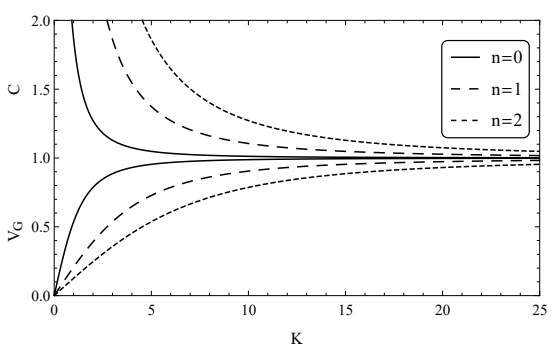

(a)

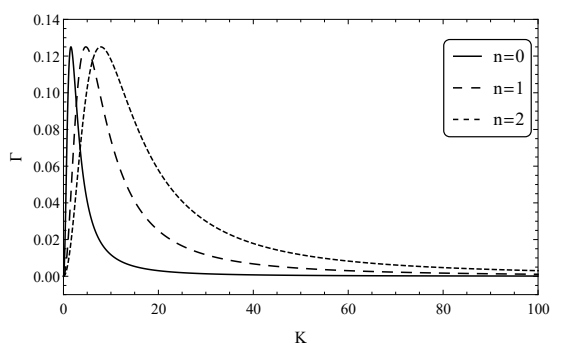

(b)

Figure 1. (a) The Variation of $C$ (upper curves) and $\mathrm{V}_{\mathrm{g}}$ (lower curves) vs $K,(\mathbf{b})$ The Variation of $\Gamma$ vs $K$ for the first three branches of the dispersion relation $(3.21)$.

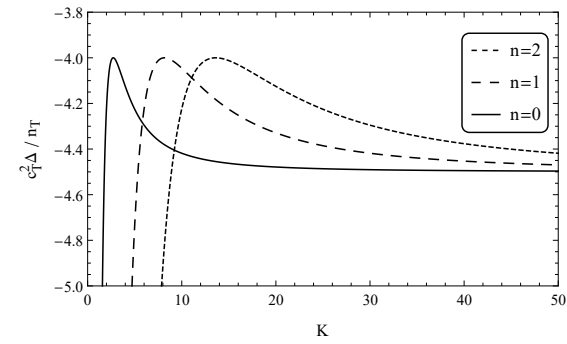

(a)

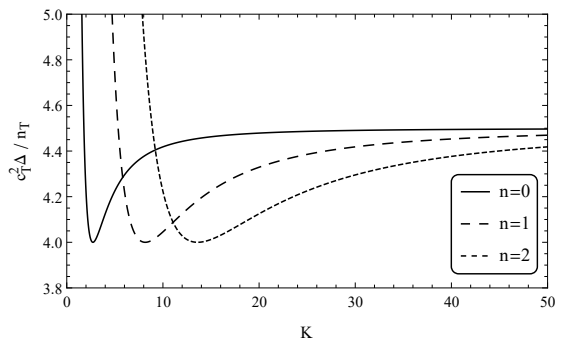

(b)

Figure 2. (a) The Variation of $\Delta c_{T}^{2} / n_{T}$ vs $K$ for a hardening layer, (b) The Variation of $\Delta c_{T}^{2} / n_{T}$ vs $K$ for a softening layer, for the first three branches of the dispersion relation (3.21).

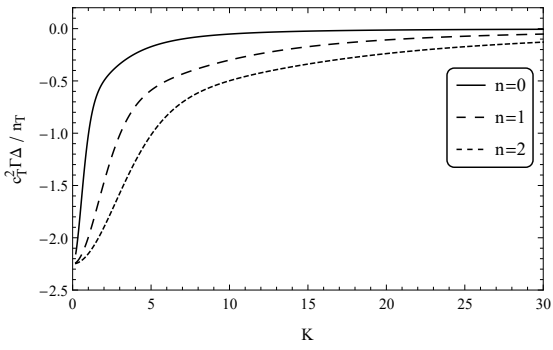

(a)

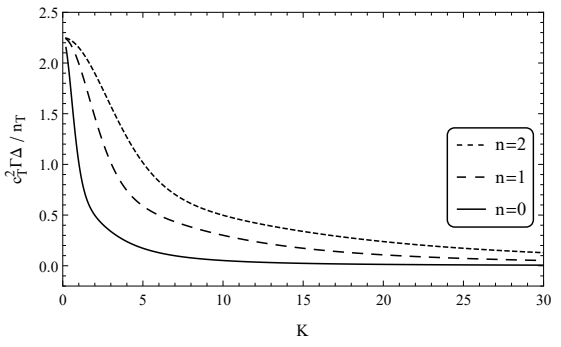

(b)

Figure 3. (a) The Variation of $\Gamma \Delta c_{T}^{2} / n_{T}$ vs $K$ for a hardening layer, (b) The Variation of $\Gamma \Delta c_{T}^{2} / n_{T}$ vs $K$ for a softening layer, for the first three branches of the dispersion relation (3.21). 


\section{References}

[1] Ewing, W.M., Jardetsky, W.S., Press, F. Elastic Waves in Layered Media, McGraw-Hill, New York, 1957.

[2] Love, A. E. H. Some Problems of Geodynamics, Cambridge University Press, Cambridge, 1911. of seismology. Cambridge University Press, Cambridge, 1963.

[3] Achenbach, J.D. Wave Propagation in Elastic Solids, North Holland Publishing Co., Amsterdam, 1973.

[4] Graf,K.F. Wave Motion in Elastic Solids, Clarendon Press, Oxford, 1975.

[5] Farnell, G.W. Types and properties of surface waves, In: A.A. Oliner (Ed.), Acoustic Surface Waves, pp. 13-60, Springer, Berlin, 1978.

[6] Maugin, G.A. Elastic surface waves with transverse horizontal polarization, In: Hutchinson, J.W. (Ed.) Advances in Applied Mechanics. 23, pp. 373-434. Academic Press, New York, 1983.

[7] Parker, D.F., Maugin, G.A. (Eds.). Recent Developments in Surface Acoustic Waves, Springer Series on Wave Phenomena, Vol. 7., Springer-Verlag, Berlin, 1988.

[8] Maugin, G.A. Physical and mathematical models of nonlinear waves in solids, In: Jeffrey, A., Engelbrecht, J. (Eds.) Nonlinear Waves in Solids, International Centre for Mechanical Sciences, Course and Lectures-No.341, pp. 109-233, Springer-Verlag, New York, 1994.

[9] Parker, D.F. Nonlinear surface acoustic waves and waves on stratified media, In: Jeffrey, A., Engelbrecht, J. (Eds.) Nonlinear Waves in Solids, International Centre for Mechanical Sciences, Course and Lectures-No.341, pp. 289-347. Springer-Verlag, New York, 1994.

[10] Mayer, A.P. Surface Acoustic waves in nonlinear elastic media, Physics Reports, 256, 4-5, 1995.

[11] Norris, A. Finite Amplitude Waves in Solids, In: M.F. Hamilton and D.T. Blackstock (Eds.), Nonlinear Acoustics, Chap. 9, pp.263-277. Academic Press, San Diego, 1998.

[12] Porubov, A.V. Amplification of Nonlinear Strain Waves in Solids, World Scientific, Singapore, 2003.

[13] Bataille K., Lund, F. Nonlinear waves in elastic media, Physica D 6, pp. 95-104, 1982.

[14] Teymur, M. Nonlinear modulation of Love waves in a compressible hyperelastic layered half space, Int. J. Eng. Sci. 26, 907-927, 1988.

[15] Maugin, G.A., Hadouaj, H. Solitary surface transverse waves on an elastic substrate coated with a thin film, Phy. Rev. B. 44 (3), 1266-1280, 1991.

[16] Teymur, M., Demirci, A., Ahmetolan, S. Propagation of surface SH waves on a half space covered by a nonlinear thin layer, Int. J. Eng. Sci. 85, 150-162, 2014.

[17] Ahmetolan, S., Teymur, M. Non-linear modulation of $S H$ waves in a two-layered plate and formation of surface SH waves, Int. J. Non Linear Mech. 38, 1237-1250, 2003.

[18] Ahmetolan, S., Teymur, M. Nonlinear modulation of $S H$ waves in an incompressible hyperelastic plate, Z. angew. Math. Phy. 58, 457-474, 2007.

[19] Teymur, M. Small but finite amplitude waves in a two-layered incompressible elastic medium, Int. J. Eng. Sci., 34, 227-241, 1996.

[20] Ferreira, E.R., Boulanger, Ph. Large Amplitude Love Waves, Q. Jl. Mech. Appl. Math. 61 (3), 353-371, 2008.

[21] Pucci, E., Saccomandi, G. Secondary Motions Associated With Anti-Plane Shear in Nonlinear Isotropic Elasticity, Q. Jl. Mech. Appl. Math. 66, 221-239, 2013.

[22] Kramer, S.L. Geotechnical Earthquake Engineering, Prentice Hall, New Jersey, 1996.

[23] Eringen, A.C., Suhubi, E.S. Elastodynamics, Vol. I. Academic Press, New York, 1974.

[24] Saccomandi,G., Ogden, R.W. (Eds.)Mechanics and Thermomechanics of Rubberlike Solids, CISM Lecture Notes 452, Springer, New York, 2004.

[25] Carroll, M.M. Some results on finite amplitude elastic waves, Acta Mech., 3, 167-181, 1967.

[26] Pucci,E., Saccomandi,G. The Anti-Plane Shear Problem in Nonlinear Elasticity-Revisited, J. Elast., 113, 167-177, 2013.

[27] Rogers, C., Saccomandi,G., Vergori, L. Carroll-type deformations in nonlinear elastodynamics, J. Phys. A: Math. Theor. 47, 205204, (17pp), 2014.

[28] Jeffrey, A., Kawahara, T. Asymptotic Methods in Nonlinear Wave Theory, Pitman, Boston, 1981. 
[29] Hudson, J.A. Love waves in a heterogeneous medium, R. Astr. Soc. The Geophys. Jou. 6, 131-147, 1962

[30] Dodd, R.K, Eilbeck J.C., Gibbon, J.D., Morris, H.C. Solitons and Nonlinear Wave Equations, Academic Press, London, 1982.

[31] Ablowitz, M.J., Clarkson, P.A. Solitons, Nonlinear Evolution Equations and Inverse Scattering, Cambridge University Press, Cambridge, 1991.

[32] Peregrine, D.H. Water Waves, Nonlinear Schrödinger Equations and Their Solutions, J. Aust. Math. Soc. Ser. B. 25, 16-43, 1983.

[33] Engin, H., Askar A. Cakmak, A.S. An Analytic Method for Strong motion Studies in Layered Media, Int. J.Non Linear Mech. 16, 165-186, 1981.

[34] Dimitriu, P.P. Experiments on Nonlinear Effects in Ground-Surface Vibration, In Parker, D.F., Maugin, G.A. (Eds.), Recent Developments in Surface Acoustic Waves, pp.72-78, Springer Series on Wave Phenomena, Vol. 7. Springer-Verlag, Berlin, 1988. 
